\title{
SEPSE COMO MOTIVO DE MORBIDADE HOSPITALAR: ANÁLISE HISTÓRICA NO PARÁ DE 2015-2019
}

\author{
SEPSIS AS A REASON FOR HOSPITAL MORBITY: HISTORICAL ANALYSIS IN PARÁ 2015-2019
}

\section{LA SEPSIS COMO MOTIVO DE MORBIDAD HOSPITALARIA: ANÁLISIS HISTÓRICO EN PARÁ 2015-2019}

\author{
João Victor Farias de Oliveira1; Stefani de Lima Carvalho²; Gabriela Andrelo Lima da Rocha;
}

Tereza Cristina de Brito Azevedo ${ }^{3}$

Submetido: $27 / 08 / 2020$

Aprovado: $26 / 10 / 2020$

\section{RESUMO}

Introdução: Sepse corresponde ao conjunto de manifestações graves no organismo, produzidas por uma resposta inflamatória do organismo inadequada, a proporção da infecção, causando falência em órgãos vitais. Objetivo: Analisar os índices de internações por sepse no Pará de 2015-2019. Método: Estudo retrospectivo, que utilizou como base de dados o DATASUS. Coletou-se as informações relevantes sobre as internações, bem como o número de óbitos. Resultados: Entre os resultados, destaca-se que, no período, registraram-se 15.987 pacientes com sepse. $30 \%$ ocorreu na população infanto-juvenil, enquanto que os idosos atingiram $38 \%$ das internações pela doença. Quanto ao sexo, $53 \%$ e $47 \%$ dos casos aconteceram em homens e mulheres, respectivamente. O custo total foi de $\mathrm{R} \$ 47.785 .278,89$ aos hospitais públicos, com média de internação de 11 dias. Observou-se ainda uma taxa de letalidade de 34,6\%. Conclusão: A morbidade por sepse apresenta números elevados. A letalidade é alta, demandando precaução com a prevenção e diagnóstico precoce.

DESCRITORES: Sepse; Morbidade; Epidemiologia.

\begin{abstract}
Introduction: Sepsis corresponds to the set of serious manifestations in the organism produced by an inadequate inflammatory response of the organism in proportion to the infection, causing failure in vital organs. Objective: To analyze the 2015-2019 hospitalization rates for sepsis in Pará. Method: Retrospective study, using DATASUS as a database. Relevant information about hospitalizations was collected, as well as the number of deaths. Results: Among the results, it is highlighted that, in the period, 15,987 patients with sepsis were registered. $30 \%$ occurred in the child and youth population, while the elderly reached $38 \%$ of hospitalizations for the disease. As for sex, $53 \%$ and $47 \%$ of cases occurred in men and women, respectively. The total cost was $R \$ 47,785,278.89$ to public hospitals, with an average hospital stay of 11 days. A lethality rate of $34.6 \%$ was also observed. Conclusion: Sepsis morbidity has high numbers. Lethality is high, demanding precaution with prevention and early diagnosis.
\end{abstract}

KEYWORDS: Sepsis; Morbidity; Epidemiology.

\section{RESUMEN}

Introducción: La sepsis corresponde al conjunto de manifestaciones graves en el organismo producidas por una respuesta inflamatoria inadecuada del organismo en proporción a la infección, provocando insuficiencia en órganos

\footnotetext{
${ }^{1}$ Acadêmico (a) de Medicina, Universidade Federal do Pará, Belém, Pará, Brasil; ORCID: 0000-0002-3778-5855 e-mail: farias. joaovictor@hotmail.com ORCID: 0000-0002-4436-7563

${ }^{2}$ Faculdade de Nutrição, Universidade Federal do Pará, Belém, Pará, Brasil; ORCID: /0000-0002-6040-3069

${ }^{3}$ Setor de Hemotransfusão, Hospital Universitário João de Barros Barreto, Belém, Pará, Brasil; ORCID: 0000-0002-9352-326X;
} 
vitales. Objetivo: Analizar las tasas de hospitalización por sepsis 2015-2019 en Pará. Método: Estudio retrospectivo, utilizando DATASUS como base de datos. Se recopiló información relevante sobre las hospitalizaciones, así como el número de muertes. Resultados: Entre los resultados, se destaca que, en el período, se registraron 15.987 pacientes con sepsis. El 30\% ocurrió en la población infantil y juvenil, mientras que los ancianos alcanzaron el 38\% de las hospitalizaciones por la enfermedad. En cuanto al sexo, el $53 \%$ y el $47 \%$ de los casos ocurrieron en hombres y mujeres, respectivamente. El costo total fue de $R$ \$ $47.785 .278,89$ para los hospitales públicos, con una estancia hospitalaria promedio de 11 días. También se observó una tasa de letalidad del 34,6\%. Conclusión: la morbilidad por sepsis tiene cifras elevadas. La letalidad es alta, lo que exige precaución con prevención y diagnóstico precoz.

DESCRIPTORES: Sepsis; Morbilidad; Epidemiología. 


\section{INTRODUÇÃO}

Sepse corresponde ao conjunto de manifestações graves em todo o organismo produzidas por uma resposta inflamatória do organismo inadequada a proporção da infecção, causando falência em órgãos vitais 1 . Por isso, a identificação da origem da infecção é fundamental para o estabelecimento da etiologia de um quadro de sepse e constitui um importante passo para escolha do tratamento mais adequado.

A estimativa é que seja de cerca de 15 a 17 milhões o número de pacientes com sepse por ano no mundo, e que, parte desses, faça parte das mais de 5 milhões de mortes anualmente. Vários fatores contribuem para esses números, como o aumento da população e da expectativa de vida, que subiu de 65,3 anos, em 1990, para 71,5 anos, em 2013 , tornando maior a população suscetível, ou seja, aquelas com mais idosas, portadoras de doenças crônicas e que possuem imunossupressão2.

Os profissionais que trabalham em Unidade de Terapia Intensiva (UTI), têm como uma de suas principais atribuições o atendimento inicial de pacientes sépticos, o diagnóstico precoce de casos e a otimização do tratamento. Deve existir uma equipe multidisciplinar para atuar no reconhecimento dos pacientes com fatores de risco, além de estabelecer medidas preventivas e protocolos assistenciais que aperfeiçoem o atendimento e garantam o controle e prevenção da evolução da doença para formas mais graves e/ou complicações fatais3.

Com isso, fica claro que é de grande importância ter conhecimento sobre a epidemiologia da sepse e, a partir disso, tomar as medidas cabíveis de prevenção, diagnóstico e tratamento precoce, já que, sem a adequada assistência, o prognóstico dos pacientes se torna bastante reservado. Portanto, o presente estudo teve como objetivo analisar os índices de internações por sepse no Pará de 2015-2019 e conhecer seus custos, óbitos e perfil dos pacientes.

\section{MÉTODO}

Estudo retrospectivo, que utilizou como base de dados o site do DATASUS. Coletou-se o número e município das internações, idade dos pacientes, custo e média de internações, bem como o número de óbitos por sepse, de janeiro de 2015 a dezembro de 2019, no estado do Pará. Os dados foram tabulados em planilha do aplicativo Microsoft Office Excel 2019 e esse trabalho foi escrito no aplicativo da mesma empresa Word 2019.

\section{RESULTADOS}

No período, registraram-se 15.987 pacientes com sepse, sendo 2018 o ano com maior número de casos, 3.812. A maioria dos casos ocorreu na região metropolitana (5.542) e destes, 3.844 foram em Belém. Aconteceram 4.911 (30\%) internações da população infanto-juvenil ( 0 a 19 anos), enquanto que os adultos (20 aos 60 anos) apresentaram a enfermidade 5.039 vezes (32\%), cerca de mil casos a menos que os idosos (maiores que 60 anos), que atingiram 6.037 (38\%) internações pela doença. Isoladamente, as mais acometidas foram as crianças com menos de 1 ano, com 3.061 casos (19\%), seguidas dos pacientes com 70 a 79 anos, com 2.183 internações $(13,6 \%)$.

Quanto ao sexo, $53 \%$ e $47 \%$ dos casos aconteceram em homens e mulheres, respectivamente. Essas internações geraram custo total de $\mathrm{R} \$ 47.785 .278,89$ aos hospitais públicos, com média de internação de 11 dias. Também foram relatados 5.538 óbitos, causando uma taxa de letalidade de $34,6 \%$, que ainda é mais expressiva nos pacientes com 70-79 anos, com letalidade de $51 \%$.

\section{DISCUSSÃO}

A sepse configura como uma disfunção de alto impacto econômico e social. De acordo com resultados de Liang et al.4 nos Estados Unidos, ela foi a condição mais cara tratada em hospitais totalizando $\$ 38$,2 bilhões com um percentual de $8,8 \%$ dos custos agregados para todas as internações no ano de 2017. A sepse apareceu à frente de outras internações de alto custo como osteoartrite que configura como a segunda condição mais dispendiosa ( $\$ 19,9$ bilhões, ou $4,6 \%$ ); recém-nascidos (terceira colocação - $\$ 16,0$ bilhões, ou 3,7\%) e infarto agudo do miocárdio (quarta colocação - $\$ 14,3$ bilhões ou $3,3 \%)$.

De acordo com este estudo que avaliou os gastos da rede pública no período de 5 anos (2015 a 2019), o total de custo notificado pelo DATASUS foi de R\$ 47.785.278,89. A comparação de despesas relacionadas à sepse entre os estudos possui limitações de estudo, as estimativas de custo variam conforme o método de cálculo, a população examinada e o protocolo da instituição5,6. No Brasil, Silva et al.7 levantaram o custo de internação por sepse de acordo com as regiões do país 
durante os anos de 2015 e 2016. A média diária de custos por paciente internado por sepse foi menor no Norte $(R \$ 292,54)$ e maior no Nordeste ( $R \$ 320,70)$.

A sepse não é apenas um problema de saúde pública e seu enfrentamento decorre da ação de diversos setores públicos8. Nos países subdesenvolvidos, a abordagem da sepse como questão pública é ainda mais desafiadora. Situações como a pobreza, corrupção política, iniquidade no acesso à saúde e redução nos recursos humanos e terapêuticos impactam na epidemiologia da doença. A disparidade de financiamento na saúde entre países desenvolvidos e subdesenvolvidos exacerbam as dificuldades para reduzir as estatísticas da sepse. De acordo com dados do Banco Mundial9, da base de dados estatísticos populacionais e de saúde nutricional, em 2017, a despesa com saúde per capita no Reino Unido foi de $\$ 3.858,7$ em comparação com o Brasil de $\$ 928,8$. A despesa anual per capita com saúde nos países de alta renda é de $\$ 5.226$ e nos países de baixa renda é de $\$ 266$. O enfrentamento da sepse requer esforços de atuação na desigualdade econômica, nos determinantes sociais de saúde e na infraestrutura da saúde pública8.

Em relação à faixa etária, em nossa análise as mais incidentes foram em menores de 1 ano (19\%) e em 70 a 79 anos (13,6\%), a letalidade média foi de $34,6 \%$ e maior na faixa de 70 a 79 anos com $51 \%$. Essa associação com a idade é comparável com o estudo 10 que descreveu uma relação diretamente proporcional entre a idade do paciente e a letalidade apresentando como resultado uma chance de morrer por sepse de 1,036 vezes maior para cada mudança de 1 ano de idade.

No tempo de internação, a média foi de 11 dias. Esse resultado é compatível com um estudo na região norte, em que a média em 2016 foi de 11,4 dias e com outro11 que apresentou tempo médio de internação em UTI em um hospital público do Pará de 11,7 dias. Além disso, uma pesquisa10 analisou a epidemiologia das internações por sepse no período de 2006 a 2015 no Brasil e o tempo médio de internação foi de 9 dias com uma diferença entre hospitais públicos (10.3 dias) e privados (7.6 dias). A letalidade também diferiu entre as instituições, no público foi de $55,5 \%$ e no privado, de $37 \%$.

\section{CONCLUSÃO}

Desse modo, é possível notar que a morbidade por sepse no Pará apresenta números elevados, principalmente em pacientes homens, crianças menores de 1 ano e idosos, além de uma letalidade elevada, demandando precaução com a prevenção e diagnóstico precoce.

São recomendados mais estudos na área, focados em fisiopatologia, doenças de bases desencadeantes e protocolos clínicos assistenciais para se obter uma visão global do assunto, a fim de se obter uma maior recuperação e, assim, menor custo, ocupação de leitos e, como objetivo fundamental, menor mortalidade por esse agravo.

\section{REFERÊNCIAS}

1. Santos MR, Cunha CC, Ishitani LH, França EB. Mortes por sepse: causas básicas do óbito após investigação em 60 municípios do Brasil em 2017. Rev bras epidemiol. 2019;22(3):e190012.

2. Lobo SM, Rezende E, Mendes CL, Oliveira MC. Mortalidade por sepse no Brasil em um cenário real: projeto UTIs Brasileiras. Rev bras ter intensiva. 2019 Mar;31(1):1-4.

3. Borges $A C N$, Costa AL, Bezerra JB, Araújo DS, Soares MAA, Gonçalves JNA, et al. Epidemiologia e fisiopatologia da sepse: uma revisão. RSD. 2020 Jan;9(2):e187922112.

4. Liang L, Moore, B, Soni, A. National Inpatient Hospital Costs: The Most Expensive Conditions by Payer, 2017. 2020 jul. In: Healthcare Cost and Utilization Project (HCUP) Statistical Briefs, Rockville (MD): Agency for Healthcare Research and Quality (US).

5. Arefian H, Heublein S, Scherag A, Brunkhorst FM, Younis MZ, Moerer O, et al. Hospital-related cost of sepsis: A systematic review. J Infect. 2017 Feb;74(2):107117.

6. Danna DM. Hospital Costs Associated with Sepsis Compared with Other Medical Conditions. Crit Care Nurs Clin North Am. 2018 Sep;30(3):389-398.

7. Silva LMN, Carneiro RF, Oliveira LPL, Raposo LM, Oliveira TA, Sugita DM. Levantamento do custo de internação por septicemia com base em protocolo atual de manejo da doença. Rev Educ Saúde. 2019;7(1):4757. 
8. Rudd KE, Kissoon N, Limmathurotsakul D, Bory $S$, Mutahunga B, Seymour CW, et al. The global burden of sepsis: barriers and potential solutions. Crit Care. 2018 Sep;23;22(1):232.

9. THE WORLD BANK. DataBank - Health Nutrition and Population Statistics. Disponível em: https:// databank.worldbank.org/reports.aspx?source=health-nutrition-and-population-statistics

10. Quintano Neira RA, Hamacher S, Japiassú AM. Epidemiology of sepsis in Brazil: Incidence, lethality, costs, and other indicators for Brazilian Unified Health System hospitalizations from 2006 to 2015. PLoS One. 2018 Apr;13;13(4):e0195873.

11. Barros LLS, Maia CSF, Monteiro MC. Fatores de risco associados ao agravamento de sepse em pacientes em Unidade de Terapia Intensiva. Cad saúde colet. 2016 Dec;24(4):388-396. 